\title{
Cultura Maker. Dispositivos, Prótesis Robóticas y Programación Visual en Arquitectura y Diseño para eficiencia energética.
}

\author{
Culture Maker. Devices, Prostheses Robotics and Visual Programming in Architecture and \\ Design for energy efficiency.
}

\author{
Chiarella, Mauro \\ CONICET/Universidad Nacional del Litoral \\ Argentina \\ chiarell@fadu.unl.edu.ar \\ Martini, Sebastián \\ Universidad Nacional del Litoral, Argentina \\ sebamartinidis@gmail.com
}

\author{
Giraldi, Sebastián \\ Universidad Nacional del Litoral, Argentina \\ arq.sebastiangiraldi@gmail.com
}

\author{
Góngora, Nicolás \\ Universidad Nacional del Litoral, Argentina \\ nicogongora@hotmail.com \\ Picco, Camila \\ Universidad Nacional del Litoral, Argentina \\ cami_picco@hotmail.com
}

\begin{abstract}
The Maker movement is the ability to be small and at the same time world; craftsmanship and innovative; high technology and low cost. The Maker movement is doing for physical products what the open source made by the software. The Maker culture emphasizes collaborative learning and distributed cognition. Its knowledge base repository and channels of exchange of ideas and information are: web sites; social networks; the Hackerspaces and Fab-Labs. Three experiences presented with devices; prostheses robotics and CNC machines, based on logical replacement; adaptation and generation. Its authors are undergraduate and graduate fellows Industrial Design and Architecture.
\end{abstract}

Keywords: Maker culture; Prostheses Robotics; Visual Programming; Energy Efficiency; Adaptive Skin

\section{Cultura Maker}

El movimiento Maker es la habilidad de ser pequeño y al mismo tiempo mundial; artesanal e innovador; de alta tecnología y de bajo coste. El movimiento Maker está haciendo por los productos físicos lo que el código abierto hizo por el software. La cultura Maker es una extensión del DIY (do it yourself) basada en la tecnología. Los intereses comunes incluyen desarrollos en electrónica, robótica, impresión 3D y el empleo de máquinas $\mathrm{CNC}$, como así también el uso de herramientas clásicas como el trabajo de herrería, tornería, carpintería y las tradicionales artes y oficios. La cultura Maker enfatiza el aprendizaje colaborativo y la cognición distribuida. La interacción en la comunidad y el intercambio de conocimientos se haya mediado por las tecnologías de trabajo en red. Los sitios web y las redes sociales son la base del repositorio de conocimientos y el canal central del intercambio de ideas e información, hibridado por encuentros físicos en espacios de intercambio social como los Hackerspaces y los Fab Lab. Impulsados principalmente por la llegada de la impresión 3D para la fabricación de prototipos, la disminución de costos y la gran aceptación de éstas tecnologías, se fueron consolidando nuevos campos de innovación. La nube en sí es una herramienta al servicio del movimiento creador, lo que permite una mayor colaboración, flujo de trabajo digital, y la manufactura compartida mediante la descarga y distribución de archivos. Mientras tanto, el movimiento de código abierto, que se había centrado inicialmente en el software, se ha ido expandiendo en el hardware, asistido por el fácil acceso a los planos en línea y acuerdos de licencia. Bajo los conceptos enunciados se presentan 3 experiencias simples basadas en lógicas de: Sustitución; Adaptación y Generación en Dispositivos; Prótesis Robóticas y Maquinas de Control Numérico realizada por tesistas de grado y posgrado FADUUNL (Diseño Industrial y Arquitectura).

\section{Dispositivos}

Podemos definir dispositivo al aparato, mecanismo o artificio que produce determinadas acciones previstas. Dispositivo digital es un conjunto tecnológico que usa distintas unidades de un sistema de procesamiento de información. El término entrada y salida de información también refiere a la ejecución de acciones u operaciones a través de dichos mecanismos. Un dispositivo de entrada o salida puede ser cualquier tipo de unidad funcional o subsistema que forma parte de un conjunto integral, pudiendo enviar señales o procesar información para establecer distintos tipos de comunicación interna y externa. 


\section{Sustitución}

SUSTITUCIÓN de Domótica de Código Cerrado. El desarrollo de un dispositivo portátil de bajo consumo para adquisición de datos destinado al análisis climático necesitó de un código base con comandos para posibilitar la interacción del servo modelo en relación a la temperatura interior-exterior censada de un prototipo de piel dinámica para eficiencia energética (arduino, fritzing, excel).

\section{Dispositivos portátiles para diagnósticos y adquisición de datos:}

En Arquitectura, es cada vez mayor el interés por el análisis riguroso de información vinculada con las variables físicas que intervienen en los ambientes construidos. Este interés se ve potenciado por la necesidad, a nivel mundial, de optimizar las decisiones de diseño a favor del ahorro energético. Estas variables nos permiten verificar cuales son las técnicas 0 tecnologías, que pueden ser aplicadas en edificios permitiendo lograr la eficiencia energética buscada. En la oferta de mercado de sistemas de registro de datos para los fines mencionados nos encontramos con dispositivos de código cerrado, limitándose las posibilidades de interactuar con la data obtenida al sistema elegido. Solo somos usuarios del sistema, solo vemos la data final que es el resultado de varios procesos de interacción de hardware y software generalmente de "caja negra" para nosotros. Estos sistemas se pueden sintetizar bajo una organización lineal que se representaría de la siguiente manera:

\section{SENSOR_BUS DE DATOS MICROPROCESADOR BUS DE DATOS_LCD PANTALLA}

Así es la organización típica de un sistema de adquisición de datos. A la señal obtenida desde los sensores se la comunica con un microprocesador a través de un bus de datos. Lo mismo ocurre entre la información enviada desde el microcontrolador a la pantalla para que los usuarios puedan ver la información de los sensores. Pero esta información final no siempre es la real emitida por los sensores, sino que puede ser modificada por filtros de hardware o software para que los usuarios no tengan problemas al momento de interpretarla. Las técnicas de filtrados comúnmente son el PROMEDIO, FILTROS EMA, FILTROS DE SOFTWARE, PASA BAJO, PASA BANDA, RECHAZA BANDA, PASA ALTOS, hasta tomar lecturas preestablecidas en rango de tiempo, cada $X$ segundos, minutos $u$ horas. Todas estas técnicas impiden que la variable registrada por los sensores llegue fielmente a la visualización en la pantalla.

La necesidad de crear un sistema de registro de datos propios el cual permita que los usuarios se conviertan en actores del mismo, permitiéndoles controlar toda la información en bruto, es el principal objetivo en la construcción del dispositivo portátil. De esta forma, se permitiría el control de información desde la emitida por los sensores sin ningún tipo de filtrado con la posibilidad futura de programar diferentes tipos de filtrados. Todo esto según la necesidad del usuario pudiendo personalizar el dispositivo según diferentes necesidades. Nuestro objetivo es tener el control total de todas las etapas en el censado de variables para la posterior confirmación o no de nuestras conjeturas, basándonos en los resultados de las mediciones efectuadas. Esta metodología podría aumentar nuestra seguridad en las afirmaciones y especulaciones sobre cuales sistemas de ahorro de energía son más eficaz que otros en un contexto determinado.

El dispositivo de adquisición de datos fue desarrollado con tecnologías de código abierto. El dispositivo fue armado con: una placa arduino uno, un módulo de fecha y hora Rtc Ds1307, un módulo de tarjeta micro sd para guardado de datos, una regulador de voltaje Im7805, una batería de $12 \mathrm{v}$ recargable. Luego de armar el software y hardware para la recolección de datos el guardado de datos en formato txt (tarjeta sd) se realiza de forma ordenada de manera que podemos extraer los mismos para cargarlos a planillas Excel para un correcto análisis de las variables a evaluar.

Una vez desarrollado nuestro dispositivo de adquisición de datos portátil (objetivo1), se inició la manufactura de un módulo simple como prototipo a escala para ejercicios de análisis de datos (objetivo 2). Éste es un marco de madera recubierto en sus caras con membranas de acetato transparente en las cuales se le realizaron perforaciones obteniendo aperturas variables para el intercambio de aire interior-exterior. En el lado superior del marco ubicamos nuestro sistema portátil de adquisición de datos en conjunto con un servo que es el mecanismo encargado de realizar el movimiento para la apertura de la piel móvil. Los módulos electrónicos utilizados son: 1 Arduino Uno; 1 Modulo RTC (incorpora datos de fecha y hora en las mediciones); 1 Modulo de tarjeta Micro SD (guardado de información); 2 Sensores de temperatura DHT11 (uno para el sensado de temperatura y humedad exterior y otro para el interior de la caja prototipo); 1 Servo motor (para el tensado sobre el acetato que actúa como piel móvil). En lo que refiere al Software para la maqueta prototipo se incluyó el código base, desarrollado para el modulo adquisición de datos pero con el agregado de los comando para hacer interactuar el servo en relación a la temperatura interior censada en la caja.

\section{Mediciones: Ganancia térmica para eficiencia energética}

Las mediciones pertinentes para el estudio de ganancias térmicas que brinda el uso de una piel responsiva para la climatización de ambientes internos, se realiza mediante la disposición del prototipo a la exposición solar directa con diferentes condiciones (apertura: nula y regulada por programación de sensor electrónico; material revestimiento: cerámica oscura y madera clara) muestreo de 15 minutos durante 24 hs en condiciones climáticas similares. Mediciones básicas: Humedad y Temperatura Interior prototipo; Humedad y Temperatura Exterior ambiente.

El primer test se realiza con el volumen completamente cerrado para evaluar la incidencia solar máxima dentro de la caja. Para acercar la medición de la maqueta a la realidad de la escala $1: 1$, se procedió a cubrir el piso, plano interno inferior, con distintos tonos de cerámica. Esta decisión fue tomada para evaluar los distintos comportamientos que pueden tener los materiales de revestimiento interior, dependiendo su color. La información recopilada desde nuestra adquisición de datos es almacenada en formato txt, un formato de fácil manejo para el armado de hojas de cálculos de Excel para la visualización gráfica de las 
mediciones de temperaturas y humedad, tanto exterior como exterior de la caja. Como podemos apreciar en el estudio gráfico de la mediciones podemos encontrar una diferencia máxima de temperatura entre interior y exterior de unos $17^{\mathrm{a}} \mathrm{C}$, en los momentos picos de exposición solar que van desde las $13 \mathrm{hs}$ a las $18 \mathrm{hs}$ en días agobiantes del verano santafesino (región Litoral-Centro Argentina). En lo que respecta a la humedad podemos ver qué ocurre un fenómeno constante de efecto inverso con la temperatura actual del momento, Esto quiere decir que a medida que incrementa la temperatura la humedad decremento, dándose así los picos de humedad por las noches. Entre las $2 \mathrm{hs}$ y 7 hs podemos ver el fenómeno de punto de rocío en el cual la humedad exterior tiende a pasar la barrera de los 90\% (Figura1, gráfico A, linea verde), y la humedad interior (gráfico A, línea azul) tiende a no seguir el mismo camino, al estar protegida dentro de la maqueta del efecto del rocío.

En el segundo caso nos dedicamos a realizar mediciones de temperatura y humedad en la maqueta, pero con la aplicación de la piel móvil. Este sistema permite crear aperturas programadas para controlar la temperatura interior del módulo, con la utilización de poca energía. Como podemos ver en el gráfico $\mathrm{B}$, la medición que más ha variado es la de temperatura interior del módulo. Si consultamos la programación utilizada en el software, podemos ver que el un efecto de abrir-cerrar-abrir-cerrar en el sistema. Volviendo a lo observado en la temperatura interior, el mecanismo de piel móvil ha permitido una mejor ventilación del módulo permitiendo que la temperatura interior no exceda la temperatura ambiente exterior durante todas las horas del día, pero también donde es más notable, en horarios picos de radiación solar.

En el tercer caso (Figura 1, Gráfico $\mathrm{C}$ ) hemos quitado la cerámica oscura dentro del volumen de la maqueta. El piso, en este análisis será de la misma madera que conforma el marco. Según lo observado en el estudio, los resultados son muy similares a la prueba realizada en el test 2 . Se puede apreciar una pendiente más suave para el aumento de la temperatura interior del módulo, entre las $9 \mathrm{hs}$ y $12 \mathrm{hs}$. Esto es debido a que al eliminar la cerámica en el piso, se ha eliminado el material capaz se absorber de manera más rápida la radiación solar y transformarla en calor. Un efecto parecido puede observarse luego de la caída del sol, se presenta una caída más marcada de la temperatura interior del volumen. El piso al ser de madera, no tiene las mismas características térmicas que la cerámica, por lo cual pierde el calor de manera más rápida. El ambiente interno se enfría más rápido. Esta experiencia se ha puesto a prueba finalmente a escala 1:1 en un vivero de 250m3 (María Juana, Pcia. de Santa Fe), encontrando curvas similares a las mediciones realizadas en
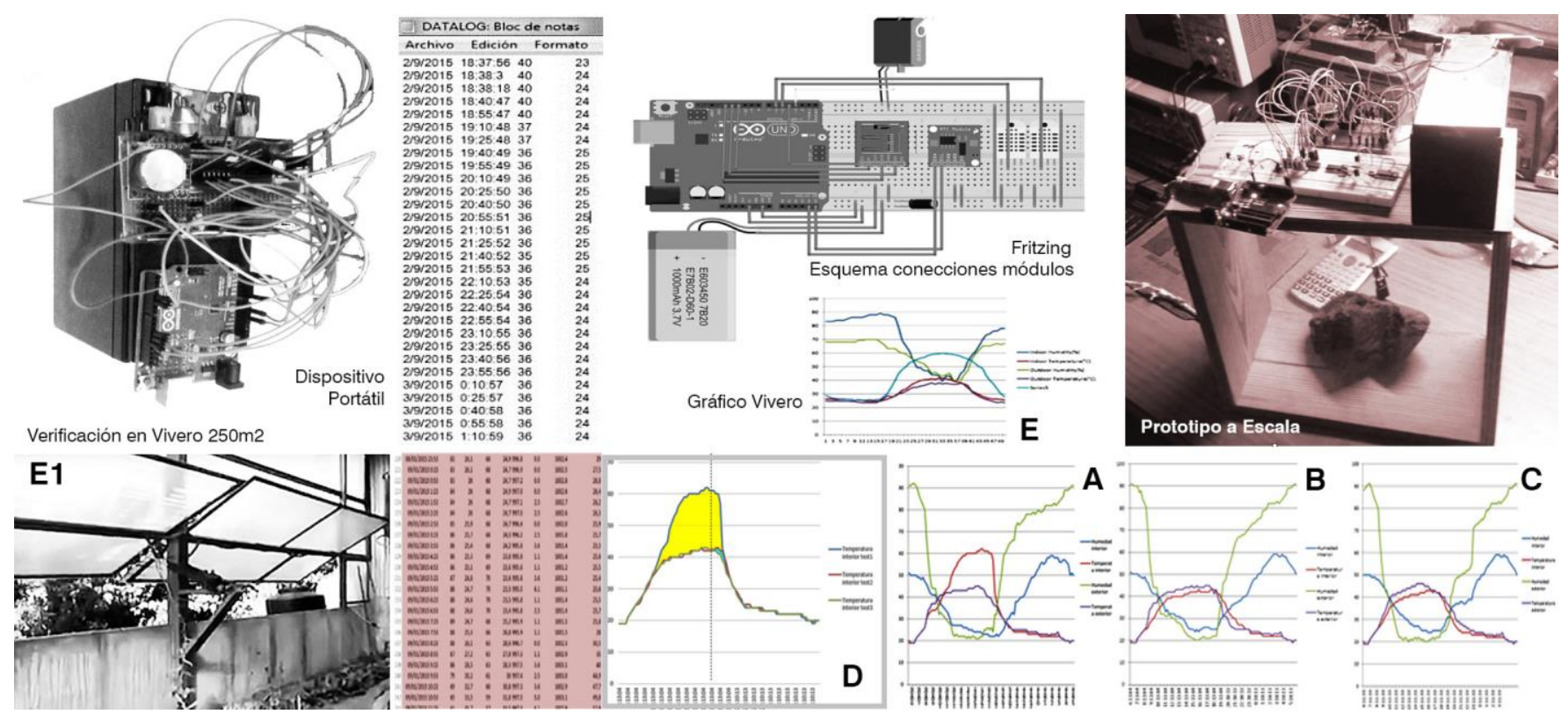

A :

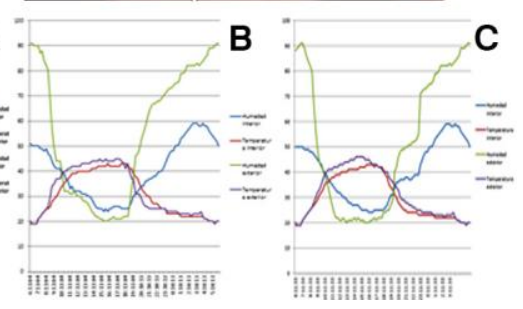

Figura 1: Dispositivo portátil para diagnóstico y adquisición de datos (arduino, fritzing, excel)

servo abre la piel móvil cuando cense igual o más de $28^{\circ} \mathrm{C}$ de temperatura interior y por efecto contrario la piel móvil cerrara cuando el Sistema este censando $25^{\circ} \mathrm{C}$ de temperatura interior. El desfase de temperatura $(>=28 ;<=25)$ adoptado es para que el sistema no presente comportamiento no deseado al realizar las actuaciones de los servos. Comprobamos que si programamos abrir y cerrar pieles a niveles consecutivos de temperatura, (ej. $25^{\circ}$ y $26^{\circ}$ ), el ingreso rápido de aire renovado hace salir al sensor de la lectura actual y lo hace volver a lecturas anteriores, impulsando al sistema a tomar la decisión anterior. Esto, manifestado en el sistema en la observación de la maqueta prototipo (Figura 1, gráfico E y E1).

\section{Resultados pieles dinámicas y mediciones}

Hemos desarrollado un sistema de bajo consumo; de código abierto; de bajo costo y portátil. Este sistema puede ser utilizado en aplicaciones a nivel maqueta o aplicaciones a escala real. En cuanto a los resultados obtenidos en los tres test, podemos observar una importante de regulación de temperatura interior produciendo ventilaciones que permitan el intercambio de aire con el exterior. 
La zona marcada en amarillo (Figura 1 , gráfico $D$ ) es la diferencia de temperatura entre el test1 y el test2 o test3 (gráfico $A$; $B$ y $C$ respectivamente), en los horarios pico de entre 13hs y 18hs. Es altamente importante que en las construcciones arquitectónicas y más aún en las obras contemporáneas en los que el vidrio inmóvil lo envuelve todo, posean ventilaciones para el intercambio de aire con el exterior. Si no se logra esto, la energía necesaria para la climatización de los ambientes interiores será mucho mayor. Si nos basamos en una temperatura de confort interior de $25^{\circ} \mathrm{C}$, la energía necesaria para climatizar un ambiente cerrado sin ventilación con el exterior será casi el doble. Por otra parte podemos observar una zona entre las gráficas del test 1 o test 2 con la curva del test3, pintada en color turquesa. Esta área corresponde a la pérdida marcada de temperatura al reemplazar el piso de madera por el de cerámica. Y finalmente la línea de puntos vertical es la indicación del pico máximo promedio de aumento de las curvas interiores de temperaturas. Es el momento en el cual las gráficas de temperaturas interiores comienzan la etapa de decrecimiento (gráfico D).

Si comparamos las gráficas obtenidas de un trabajo de automatización para climatización de nuestra autoría en escala 1:1, aplicado en un vivero de 250m3 (gráfico $\mathrm{E}$ ), encontramos curvas similares a las mediciones realizadas en maqueta. Lo correspondiente a Humedad relativa, no decae la misma por debajo de los $40 \%$. Factor que en las pruebas en la maqueta prototipo el límite de esta variable es mucho menor. La razón de esta diferencia se debe a que el vivero dispone de un sistema de dispersión de gotas de agua que permiten mantener la humedad interior. Además de la presencia abundante de plantas que se encargan de mantener estos niveles de vapor de agua en el aire. En cuanto a la Temperatura interior del vivero se comporta igual que la maqueta automatizada. El vivero cuenta con un sistema similar de automatizado. El gráfico $\mathrm{E}$, corresponde a la temperatura interior del vivero cuando no estaba automatizado, esto es, cerrado por completo con el $40 \%$ de paredes de material traslucido tipo policarbonato. Si comparamos esta gráfica con la prueba test1 de la maqueta prototipo (gráfico A), ambas curvas son similares demostrando la pertinencia del dispositivo desarrollado.

\section{Prótesis Robóticas}

Una prótesis robótica es un elemento artificial dotado de cierta autonomía e inteligencia capaz de realizar una función de una parte faltante del cuerpo humano. Dicha autonomía e inteligencia se logra al integrar sensores, procesadores, actuadores y complejos algoritmos de control.

\section{Adaptación}

ADAPTACIÓN contextual de Domótica de Código Cerrado. Con el objetivo de dar una respuesta integral que ponga como centro de la programación a las conductas vivenciales y cambiantes de los ocupantes de un edificio, se aplica un sistema basado en encuestas a usuarios, mandantes y fabricantes, que combina el análisis del edificio existente con un dispositivo a modo de interface de celosías dinámicas industrializadas. (arduino, grasshopper, firefly).

\section{Post-Occupancy Evaluations - POE (Encuesta de percepción usuarios CITEC)}

Muchos edificios no se llevan a cabo como estaban previsto o sufren modificaciones sobre la marcha, teniendo un impacto en los costes de funcionamiento, el usuario, el rendimiento, la salud, la seguridad y el confort. La evaluación post-ocupación (POE) es el proceso de obtención de información sobre el rendimiento de un edificio en uso. El valor de POE está siendo cada vez más reconocido, y se está convirtiendo en seguimientos obligatorios en muchos proyectos públicos. POE es valiosa en todos los sectores de la construcción, especialmente salud, educación, oficinas, comercial y vivienda, donde los malos resultados tendrán un impacto en los costes de funcionamiento, el bienestar del usuario y en la eficiencia energética edilicia. La evaluación post-ocupación realizada a los usuarios del edificio CITEC (Universidad del Bio-Bio, Concepción-Chile) en relación a las celosías automatizadas de control solar ha tenido los siguientes objetivos:

- Destacar los problemas iniciales inmediatos que pueden ser abordados y solucionados;

- Identificar las lagunas en la comunicación y la comprensión del impacto en las formas de operar de los sistemas propuestos en edificio

- La consulta de los ocupantes en relación a la comodidad y el control sobre las condiciones ambientales que inciden en la eficiencia energética edilicia

- Proporcionar lecciones que se pueden utilizar para mejorar el diseño y la contratación en proyectos futuros

\section{Prótesis Robóticas de código abierto:}

Este desarrollo parte como una aproximación a establecer herramientas de análisis y una propuesta de solución para factores de Post-Ocupación (en inglés Post-Occupancy Evaluations - POE) o sea, la manera de determinar cómo se está usando un edificio y que problemas se encuentran. La solución al problema encontrado fue el diseño y construcción de un dispositivo híbrido (prótesis robótica) que busca establecer un dialogo entre sistemas de programación open source y sistemas industriales de hardware y software cerrado.

La programación se hizo en el entorno de Grasshopper (plugin de Rhino) y utilizando Firefly que es un conjunto de herramientas que opera sobre Grasshopper. Dichas herramientas permiten conectar en tiempo real la programación digital de grasshopper con el hardware, que en este caso es un microcontrolador Arduino. Es así como la placa Arduino viene a funcionar como interface entre la programación digital y el modelo físico que son los dedos robóticos, los cuales van a operar sobre el control remoto que permite la apertura o cierre de las celosías Hunter Douglas. En cuanto a la descripción de la programación se puede describir en los siguientes pasos;

- Establece la comunicación entre el computador y el encendido de la placa Arduino

- Lee la hora del computador (laptop), y en el caso de que el dispositivo funcione en forma autónoma 


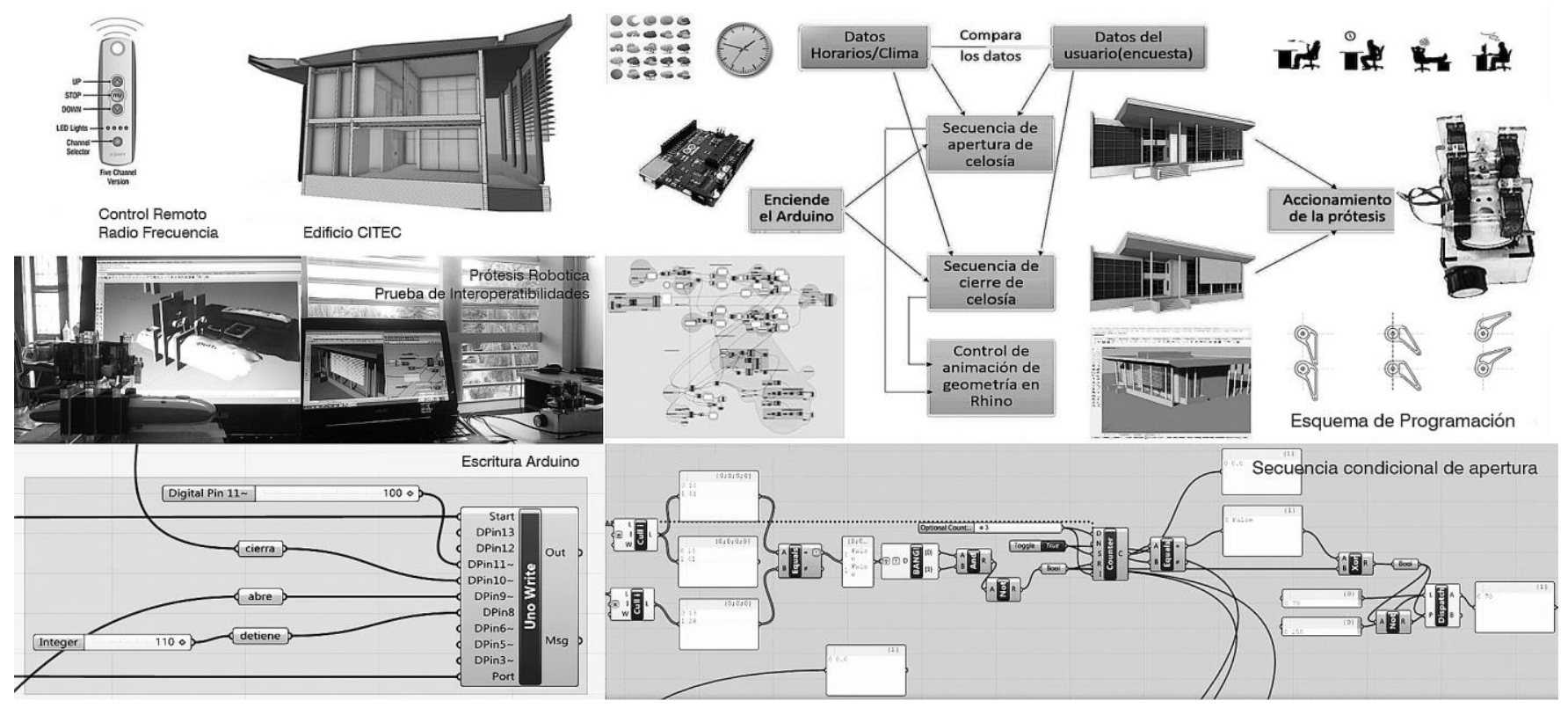

Figura 2: Prótesis Robótica de código abierto (arduino, grasshopper, firefly)

placa Arduino.

- Se establece la hora de apertura o cierre de las celosías según la preferencia de los usuarios, dato tomado a través de las encuestas.

- Se activa el paquete de códigos que mandan la orden de apertura de la celosía, parámetro True False en programación algorítmica.

- Se activa el paquete de códigos que mandan la orden de cierre la de celosía, parámetro True False en programación algorítmica.

- Se activa la animación en Rhino de lo que está sucediendo con las celosías, y es necesario que esté conectada la laptop. Esto punto es solo para monitoreo y futuras correcciones con los usuarios.

Si bien en la prueba de campo se usó una laptop conectada al Arduino para hacer las correcciones, el objetivo final es dejar el dispositivo trabajando en forma autónoma sin estar conectado a esta. Esto se puede hacer ya que placa Arduino tiene una memoria y se puede dejar grabada las ordenes de la programación y por otro lado se le tendría que agregar un reloj y una batería externa, como la que se usan para la carga de celulares. Esta batería se conecta al dispositivo a través de un cable USB. La importancia de agregar un reloj es fundamental, ya que este es el que define el momento en que los dedos robóticos deben accionar sobre el control remoto.

Con respecto al análisis post-ocupacional se basaron tanto en aspectos cuantitativos como cualitativos. En los primeros se tomaron mediciones con sensores luminotécnicos Tipo Hobbo durante una semana, en la oficina que presentaba mayores quejas de deslumbramiento por parte de los usuarios. Estos sensores miden y registran la cantidad de iluminación natural recibida cada cierta frecuencia, que en este caso era cada 15 minutos. a una planilla Excel para luego ser leída desde la programación del grasshopper. Una de las finalidades que se pensó primeramente era operar los dedos robóticos según un óptimo de iluminación natural (dato cuantitativo).

En el análisis cualitativo, se optó por realizar entrevistas a los mandantes y encuestas a los usuarios. Con los primeros, la idea era poder tener un panorama de cuáles eran las premisas de cómo debía funcionar el edificio cuando lo proyectaron, y contraponer esto con las encuestas a los usuarios para ver cómo ellos percibían su funcionamiento.

Dicha encuesta se centró en los factores lumínicos principalmente, excluyendo los factores térmicos o acústicos ya que el uso de las celosías en este caso en particular del CITEC no gravita sobre estos. Por otro lado se les consulto a los usuarios sobre la percepción que tenían de la imagen del edificio como representación de las actividades que allí se realizan (Centro de Investigación Tecnológica). Esto es debido a que este desarrollo pertenece a una tesis de maestría que se debate entre la tensión de lo simbólicoexpresivo de la arquitectura vs el desempeño funcional.

El resultado de la encuesta fue que hay un problema de deslumbramiento principalmente en una oficina del primer piso y más precisamente sobre una persona que es la que se ve más afectada. Se comprobó que dicha persona es al final la que termina operando el control remoto para abrir o cerrar la celosía según el grado de disconfort que el deslumbramiento le genere. Procesando todos estos datos, se llegó a la conclusión que el mayor grado de deslumbramiento se produce en invierno, principalmente de 9 a $10 \mathrm{hs}$ a.m. debido al azimut y altitud del Sol (Concepción, Chile) y como ingresan los rayos solares a la oficina. Este dato fue clave para la programación de los dedos robóticos, lo cuales a esa hora tienen que operar sobre el control remoto para cerrar las 
celosías. Se procedió a la manufactura de la prótesis robótica, ajustes e implementación del dispositivo propuesto.

\section{Programación Visual y Código Abierto}

La Programación Visual ha posibilitado a Diseñadores y Arquitectos intervenir sobre sus propios instrumentos proyectuales innovando en los procesos de diseño. La personalización y creación de formas de trabajo mediante scripts permite la generación de instrumentos acordes a las nuevas lógicas propuestas. Es así como la aplicación de secuencias de comandos para la productividad, la experimentación y la especulación en el diseño permite la creación de prototipos dinámicos basados en geometría generativa complementándose con programación de código abierto para la generación de máquinas de prototipado personalizadas directamente vinculadas a las mismas.

\section{Generación}

GENERACIÓN de Dobladora de alambre 3D para Prototipado Rápido. En Arquitectura y Diseño Industrial la definición morfológica y espacial se realiza generalmente mediante el armado de lineas espaciales continuas y quebradas constituyendo estructuras conceptuales o de manufactura de las mismas exploraciones. El plegado espacial-físico de materiales lineales mediante las maquinas CNC, amplía recursos proyectuales mediante la programación visual potenciando lógicas implícitas de los propios procesos de diseño (arduino, processing, grasshopper). La finalidad de esta máquina es contar con un instrumento proyectual para procesos geométricos espaciales basados en patrones lineales optimizados para eficiencia energética.

\section{Prototipo Rápido CNC (3D Bending) y Programación de Código Abierto:}

Para la construcción de la dobladora de alambre 3D se ha tomado como referencia el proyecto de PensaLabs DIWire. Mecanismos simples y piezas que se crean desde una 3D Printer constituyen el armado físico de la maquina. La información básica está compartida en línea. La complejidad mayor se encuentra en el diálogo del hardware, la placa Arduino y los programas vectoriales 3D utilizados. Sobre los avances en la programación de los mismos podemos mencionar los siguientes pasos desarrollados:

1. En principio, para obtener las figuras que se desean curvar se utiliza el programa Rhinoceros para modelar curvas trabajando con el plugin Grashopper, el cual permite hacer variantes de curvas a través del diseño paramétrico.

2. A partir de las curvas dibujadas se obtiene una nube de puntos que están en coincidencia con la línea. Desde Rhino se exportan los puntos generados anteriormente en formato "Puntos txt". El archivo resultante tiene una lista ordenada de números entre comillas, separados en tres columnas por coma. Cada columna representa a las coordenadas $X, Y$ y $\mathrm{Z}$ respectivamente.

3. Con el programa Processing se realiza el primer módulo, el cual toma las coordenadas desde el archivo que genera
Rhino y las convierte a un formato intermedio con los números sin comillas, y separados por punto y coma. Este primer módulo, en esta primera etapa, tiene una tarea muy simple (sacar comillas y reemplazar comas por punto y comas). Sin embargo, su misión, a futuro es aceptar una variedad más grande de datos de coordenadas: Por ejemplo, lista de números con o sin comillas, separadas por espacios en blanco, tabulador, coma, punto y coma, o cualquier otra serie de caracteres.

1. El segundo módulo, también realizado en Processing, tiene como misión convertir las coordenadas de la nube de puntos, en datos comprensibles por la Dobladora de Alambre (largo de alambre, ángulo de pliegue, rotación del alambre).

a. Para obtener el largo de alambre que debe salir de la máquina antes de realizar un pliegue, se toman dos puntos y se calcula la distancia entre ellos con el teorema de Pitágoras $(\mathrm{D} 2=\Delta \mathrm{X} 2+\Delta \mathrm{Y} 2+\Delta \mathrm{Z} 2)$

b. Para obtener el Ángulo de pliegue, se toman tres puntos para formar dos segmentos, y luego se calcula el ángulo entre ellos por medio del "producto escalar".

c. Para obtener el Ángulo que debe rotar el alambre, se toman cuatro puntos, para armar tres segmentos. Primero se calculan las direcciones de los vectores normales, perpendicular a los dos segmentos, al pliegue entre el primer y segundo segmento, y lo mismo para el segundo y tercer segmento. Ya con las dos direcciones normales, se calcula el ángulo entre las mismas. Ese ángulo es el que deberá rotar el alambre antes de realizar el próximo pliegue.

d. Este módulo genera un archivo con los tres parámetros calculados (largo, ángulo de pliegue, ángulo de rotación). Este módulo puede incorporar la posibilidad de invertir el orden de las secuencias, como una opción más de la dobladora. Esta opción se agregaría al principio de este módulo, antes de los cálculos.

2. El último módulo de Processing es el que se comunica con la placa de Arduino y está compuesto por dos sub módulos:

a. Al conectar la computadora con la dobladora, el primer módulo es el encargado de iniciar el diálogo. Cuando recibe respuesta, pasa al siguiente módulo. A futuro, este módulo enviará comandos en varias velocidades, y por varios puertos hasta que llegue la respuesta de la dobladora, indicando que se logró la comunicación. Para que dicha comunicación entre la computadora y la placa de Arduino sea posible, deben estar configuradas a la misma velocidad. El escaneo puertos y velocidades serviría para que la máquina sea autoconfigurable.

b. El segundo módulo interactúa con la dobladora, y responde a las consultas de datos.

3. El programa de la placa Arduino, al iniciarse, queda a la espera de un comando, el cual es un "1", que indica a la dobladora que comienza el proceso. Una vez recibido el comando de inicialización, la dobladora envía una respuesta de "ok", y pasa al siguiente módulo. 
SIGraDi 2016, XX Congreso de la Sociedad Ibero-americana de Gráfica Digital 9-11, November, 2016 - Buenos Aires, Argentina

4. En el próximo módulo, la dobladora envía la consulta de parámetro, y al recibir la respuesta envía la siguiente consulta de parámetros. Al completar los tres parámetros, empieza con los tres parámetros siguientes. El tamaño de los paquetes de parámetros dependerá de la memoria disponible en la placa de Arduino y es un valor que se configura en el módulo del programa de Arduino. Al completar la carga del paquete de parámetros, pasa al módulo siguiente.

5. El módulo que ejecuta la secuencia, empieza a recorrer los parámetros almacenados, y los envía al módulo de salida. Una vez terminada la secuencia completa de parámetros, vuelve al módulo anterior para pedir el paquete de parámetros siguientes.

6. Finalmente, el último módulo recibe la orden del módulo anterior, y convierte cada parámetro en cantidades de pulsos de motor, y los envía a la salida. Si el motor de avance del alambre necesita hacer 5 pasos para que el avance $1 \mathrm{~mm}$. Este módulo calcula el parámetro de "largo de segmento $=125 \mathrm{~mm}$ " $\times 5$ pasos $=625$ pasos, y lo envía al hardware. (del mismo modo ocurre con el motor de plegado y rotación.). De esta manera quedaría programada la máquina para poder curvar los distintos modelos que se diseñen y compartir su aventura con quien esté interesado. Según Anderson durante la última década se trató de buscar formas de crear, inventar y trabajar juntos en la web. En la próxima década estos aprendizajes se concentrarán en la construcción y manufactura colectiva de objetos del mundo físico. El movimiento Maker está haciendo por los productos físicos lo que el código abierto hizo por el software.

Durante décadas, debido a los conocimientos, el equipamiento y los costos implicados en producir objetos a gran escala, la fabricación ha sido sobre todo una cuestión de grandes empresas y profesionales muy bien formados. Hoy en día las empresas nacen en dormitorios de estudiantes y se hacen grandes antes de que sus fundadores se gradúen. Basándose en la fabricación digital de alta tecnología, es posible que individuos emprendedores puedan utilizar a las grandes empresas a voluntad para realizar lo que deseen. En la variabilidad está el desafío y la impronta del Diseño personalizado.

El aprendizaje colaborativo y la cognición distribuida también cuestionan el mito de la originalidad exclusiva de autor y su obra. No es necesario crear algo desde cero o tener una idea única y original. Es posible participar en la mejora colectiva de

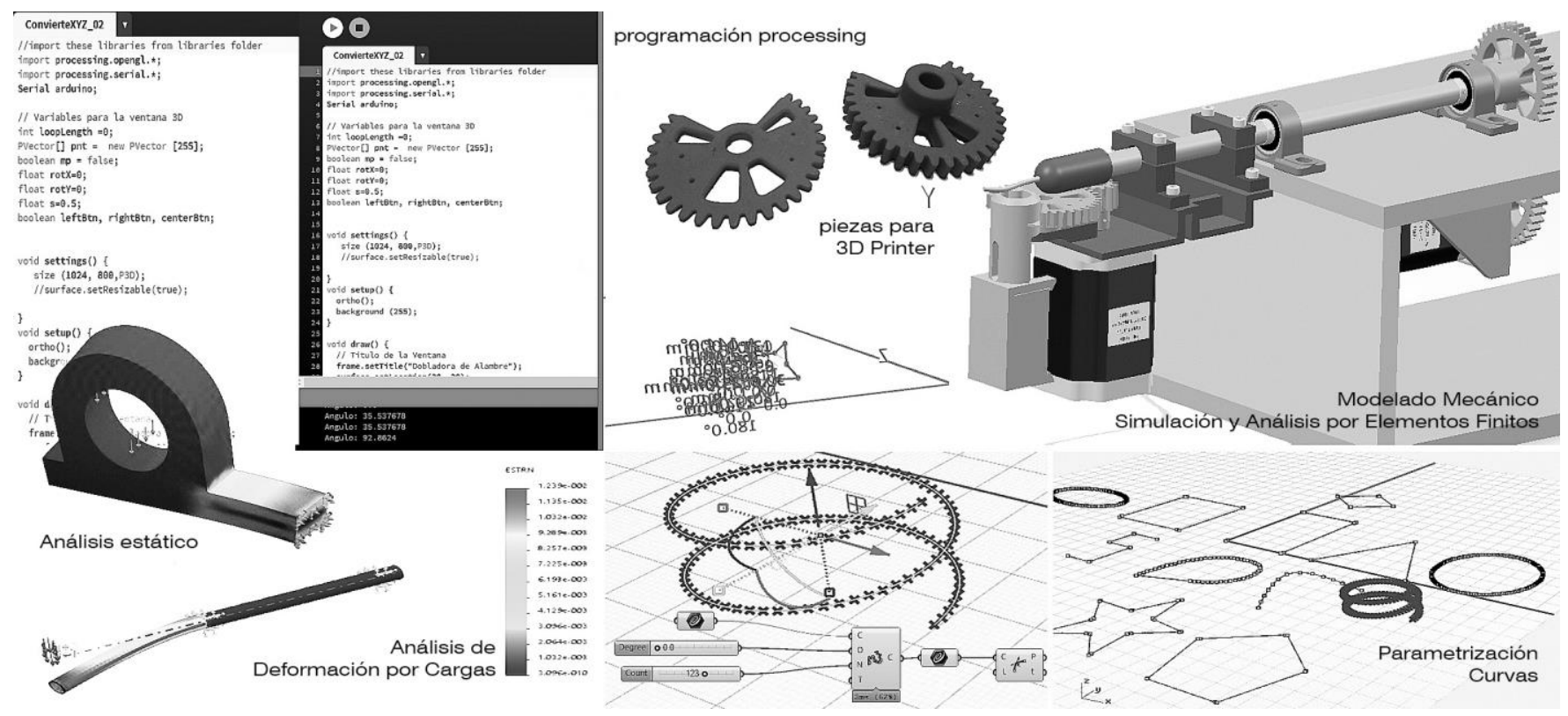

Figura 3: Diseño y Manufactura de máquina de Prototipado Rápido CNC: 3D Bending (SolidWorks; Processing; Grasshopper; Arduino)

estaría resuelta la interoperatibilidad de software.

\section{Crowdthinking}

Participar de experiencias vinculadas a la Cultura Maker permite una importante salida para la exploración personal, aumenta la comprensión de cómo los medios electrónicos funcionan y compromete a los individuos a ser actores en una cultura que es cada vez más compleja, tecnológica y digitalizada. Permite la construcción de espacios de poder íntimos y personales interactuando con multitudes. Es el poder de una sola persona para dirigir su propia educación, encontrar su propia inspiración, moldear su propio entorno y ideas o diseños ya existentes. Los proyectos compartidos en línea inspiran a otros y son una oportunidad de colaboración infinita.

\section{De Inventor a Emprendedor}

Mientras que en el pasado los inventores independientes se veían obligados a patentar sus inventos, en el presente la tendencia es publicarlo en línea bajo licencias de código abierto. De esta forma se incentiva un modelo donde la investigación y el desarrollo no tienen altos costos posibilitando la inserción en el mercado de lotes pequeños por emprendedores incipientes. Ya no se trata de poseer los 
medios de producción, sino de alquilarlos. El diseño personalizado se revaloriza ante una manufactura por demanda y una producción artesanal mecanizada. La perfecta combinación de inventar localmente y producir mundialmente permite atender a nichos de mercado definido por una demanda real y no por una geografía determinada.

Lo que se inició como un cambio cultural en las formas de hacer y pensar colectivamente, está convirtiéndose también en un cambio económico. La mejor forma de reinventar una industria madura es abrir a las ideas de otros. Antes que una innovación de arriba hacia abajo por parte de algunas de las empresas mas grandes del mundo, comienza a verse innovaciones de abajo hacia arriba iniciadas por muchos individuos, aficionados, emprendedores y profesionales.

La financiación colectiva o micromecenazgo (Crowdfunding) es la construcción de capital de riesgo para el movimiento Maker. Nuevas herramientas de captación de capital crean nuevos inversores de acuerdo a las lógicas colectivas de los emprendedores makers. Se invierte en una idea o producto, no en una empresa. El creador sube una idea que va actualizando durante su desarrollo hasta su culminación. Los padrinos o promotores hacen consultan y sugerencias durante el proceso haciendo evolucionar el producto. En el curso de este intercambio colectivo se afianza la recaudación de dinero al mismo tiempo que se crea un grupo de apoyo al emprendimiento. Quienes lo respaldan financieramente ansían el producto no sólo porque han puesto dinero en él, sino porque se sienten copropietarios de su creación. Existen diferentes modelos apuntados a necesidades diversas, pero todos se caracterizan por la participación masiva de inversores que financian con montos reducidos pequeños proyectos de alto potencial y por el carácter arriesgado de dicha inversión.

La creciente demanda de personalización de productos con la producción a medida y en pequeñas series sin aumento de costes de producción; la adaptabilidad contextual dinámica; exploración de sistemas biomiméticos; interfases mediante creación colectiva y diseño colaborativo propone desplazamientos disciplinares. A los arquitectos los alienta a desplazarse de un mero diseñador y constructor, hacia la actuación mas estratégica como mediador dentro de una amplia red de posibilidades y nuevas incumbencias disciplinares. A los diseñadores industriales, los desafía a generar nuevos desarrollos competitivos antes la recurrente inestabilidad de la tríada, empresa-producto-mercado. La cultura Maker amplía el campo de acción de las disciplinas proyectuales al incorporar nuevas herramientas en la ideación, diagnóstico y adquisición de datos contextuales repensando procesos conocidos desafiando, inclusive, las propias incumbencias profesionales.

Bajo los conceptos enunciados se han desarrollado las tres experiencias descriptas fomentando lógicas de aprendizaje colaborativo y conocimiento distribuido entre tesistas de grado, posgrado, becarios e investigadores de las carreras de Arquitectura y Diseño Industrial. Las investigaciones y desarrollos continuarán progresando bajo el desafío de conectar los ejercicios de sustitución, adaptación y generación (dispositivos, prótesis robóticas y programación visual y código abierto) para la creación de una piel, envolvente o instalación arquitectónica que verifique mejoras sustanciales en algunas de las variables físicas que influyen en la eficiencia energética edilicia.

\section{Agradecimientos}

Al Dr. Rodrigo García Alvarado, Director del Doctorado en Arquitectura y Urbanismo (DAU, UBioBio), Braulio Gatica (FACyD-UBB); Personal Técnico y Administrativo CITECUBB; Nicolás Kakisu (FADU-UNL); Carla B. Tortul (FADUUNL); Tec. Edgardo A. Gambandé; D.I. Juan López Coronel (FADU-UBA); equipo de PensaLabs (Brooklyn, NYC). Proyecto PICT2014-3567 (M.Chiarella).

\section{Referencias}

Anderson, C. (2013). Makers: The New Industrial Revolution. Crown Business.

Burry, M. (2011). Scripting Cultures: Architectural Design and Programming. Wiley

Chiarella, M; Garcia Alvarado, R.; Martín-Pastor, A. (2016). Proyectación y Construcción Digital. En L. Muller, Reffinetti Martins, M.L. (Eds), Arquitetura e qualidade socioambiental nas cidades do Cone Sul. (Cap III, pp. 431-445). Programa CAPF-BA. (CAPES-SPU). USP-UNL. Ediciones UNL. Argentina

Chiarella, M; Raffin, A; Martini, S; Gongora, N; Bressan, F. Pieles Arquitectónicas Dinámicas. Prototipos a escala mediante prototipado rápido, microcontroladores y patrones plegados., $\mathrm{p}$. 96-100. In: Proceedings of the XVIII Conference of the Iberoamerican Society of Digital Graphics: Design in Freedom. São Paulo. 2014. 